\title{
OPTIMAL SEGMENTED LINEAR REGRESSION FOR FINANCIAL TIME SERIES SEGMENTATION
}

\author{
Chi-Jen Wu, Wei-Sheng Zeng and Jan-Ming Ho \\ Institute of Information Science, \\ Academia Sinca \\ \{cjwu\}@arbor.ee.ntu.edu.tw, \{wszeng,hoho\}@iis.sinica.edu.tw
}

\begin{abstract}
Given a financial time series data, one of the most fundamental and interesting challenges is the need to learn the stock dynamics signals in a financial time series data. A good example is to represent the time series in line segments which is often used as a pre-processing step for learning marketing signal patterns in financial computing. In this paper, we focus on the problem of computing the optimal segmentations of such time series based on segmented linear regression models. The major contribution of this paper is to define the problem of Multi-Segment Linear Regression (MSLR) of computing the optimal segmentation of a financial time series, denoted as the MSLR problem, such that the global mean square error of segmented linear regression is minimized. We present an optimum algorithm with two-level dynamic programming (DP) design and show the optimality of OMSLR algorithm. The two-level DP design of OMSLR algorithm can mitigate the complexity for searching the best trading strategies in financial markets. It runs in $\mathrm{O}\left(k n^{2}\right)$ time, where $n$ is the length of the time series sequence and $k$ is the number of non-overlapping segments that cover all data points.
\end{abstract}

Index Terms - time-series, financial signal processing, segmented linear regression, stock market signal.

\section{INTRODUCTION}

Learning investment or trading signals from financial market data is one of most fundamental and interesting research challenges in both academia and industry [1, 2]. For example, the American hedge fund, Renaissance Technologies ${ }^{1}$ has leveraged financial signal processing technologies in stock trading for a long time. However, financial time series is difficult to summarize or be represented due to its highly non-stationary nature [3]. Given a financial time series data, an initial processing step of learning the signal patterns is often to represent the time series in line segments to alleviate data uncertainty and noise [4].

In a segmentation process, a time series is divided into $k$ non-overlapping segments, and each segment is represented

\footnotetext{
${ }^{1}$ https://rentec.com
}

by a model to describe data points in the segment. The segment representation is measured using error functions depending on requirements of their applications. Actually, time series segmentation is widely for dimensionality reduction purposes in economics, engineering and science [5].

Time series segmentation has been extensively discussed in different domains and various models, which has resulted in a large number of works [6, 7]. In 1961, the first version of time series segmentation problem is reported in [8] and a dynamic programming (DP) algorithm with time complexity $\mathrm{O}\left(k n^{3}\right)$ is also described. Time series segmentation also arises in data mining applications. The article [9] gives a review on applications of segmentation methods in data mining. The methods are classified into three categories, including sliding windows, bottom-up and top-down methods. The experimental comparisons showed that the bottom-up method results in better performance than other methods.

In the past few years, a few algorithms [10, 11, 12, 13] have been proposed to reduce the time complexity of time series segmentation problem. The objective is to simplify represent of large scales time series data. The Piecewise Linear Approximation (PLA) [10] is a widely used approach for the segmentation task. Acharya et al., [12] presented nearlinear time algorithms that achieve a significant improvement compared to the DP approach on large time series. Interested reader can refer to Esling and Agon [7] who present a survey on approximation segmentation of time series. To the best our knowledge, previous approaches have not addressed the problem of optimum segmentation of financial time series. Most of them discussed segmentation methods in terms of approximation representation [10], on-line processing [11] and their time complexity [12].

In this paper, we are interested in the open question [14], how to best choose $k$, the optimal number of segments used to represent a particular time series. For financial trading strategies, $k$ is a measure of number of times of changes in market trend. It is also an indicator of how many time to trade in the market while receiving a reasonable amount of trading profits. Instead of answering the open question directly, we will start with focusing on minimizing global square error for a given 
$k$, and also derive the optimal representation of each of the $k$ segments.

Firstly, we formulate the Multi-Segment Linear Regression (MSLR) problem and define the MSLR square error as the performance index. Then, we present the Optimal MultiSegment Linear Regression (OMSLR) algorithm, the twolevel DP approach for producing the globally optimal segmentation. Finally, we show the optimality of the proposed OMSLR algorithm. The time complexity of the OMSLR algorithm is $\mathrm{O}\left(k n^{2}\right)$, where $n$ is the length of the time series and $k$ is the number of non-overlapping segments that cover all data points. To the best our knowledge, this paper is the first to investigate the global optimal segmentation problem in time series processing, especially for financial time series.

This paper is organized as follows. In Section 2 we present the formulation of segmentations as an optimization problem, named MSLR problem. In Section 3 we present the OMSLR algorithm. Some segmentation experiments are presented in Section 4 , and the results are summarized in Section 5

\section{FORMULATION OF PROBLEM MSLR}

A formal definition of the Multi-Segment Linear Regression (MSLR) problem is described in this section. Given a time series $X=\left\{x_{1}, x_{2}, \ldots, x_{n}\right\}$ and an integer $k$, the objective to MSLR problem is to partition $X$ into $k$ contiguous and non-overlapping intervals, i.e., $\left[l_{i-1}, l_{i}\right)$ and $\left[l_{k-1}, l_{k}\right]$, where $l_{0}=1, l_{k}=n, 1 \leq l_{i} \leq n, 1 \leq i \leq k-1, l_{i} \in \mathbf{N}$, such that the multi-segment linear regression square error, $\psi^{2}\left(1, n \mid \phi_{k}\left(X_{n}\right)\right)$, with respect to the $k$-segment partition $\phi_{k}\left(X_{n}\right)=\left\{1, l_{1}, \ldots, l_{k}\right\}$ is minimized. Note that $\psi^{2}\left(1, n \mid \phi_{k}\left(X_{n}\right)\right)$ is also denoted as the Global Mean Square Error of the multi-segment linear regression representation, or GMSE for short. In other words, we have

$$
\begin{aligned}
& \psi^{2}\left(X_{n} \mid \phi_{k}\left(X_{n}\right)\right) \\
& =\sum_{i=1}^{k-1} \sigma^{2}\left(l_{i-1}, l_{i}-1\right)+\sigma^{2}\left(l_{k-1}, l_{k}\right) \\
& =\psi^{2}\left(X_{l_{k-1}-1} \mid \phi_{k-1}\left(X_{l_{k-1}-1}\right)\right)+\sigma^{2}\left(l_{k-1}, l_{k}\right),
\end{aligned}
$$

where $\sigma^{2}(i, j)=\sum_{m=1}^{j}\left(x_{m}-\mu(i, j, m)\right)^{2}$ and $\left.\mu(i, j, m)\right)=$ $\beta_{i j} * m+\alpha_{i j}, i \leq m \leq j$ with $\alpha_{i j}$ and $\beta_{i j}$ being the linear regression parameters on the interval $[i, j]$ of the time series $X_{n}$, i.e., $X_{i j}=\left\{x_{i}, x_{i+1}, \ldots, x_{j}\right\}$. Thus we have $\alpha_{i j}, \beta_{i j}$ and $\sigma^{2}(i, j)$ as follows.

$$
\begin{aligned}
\alpha_{i j} & =\bar{x}_{i j}-\beta_{i j} * \bar{t}_{i j} \\
\beta_{i j} & =\frac{\sum_{m=i}^{j}\left(x_{m}-\bar{x}_{i j}\right)\left(m-\bar{t}_{i j}\right)}{\sum_{m=i}^{j}\left(m-\bar{t}_{i j}\right)^{2}} \\
\sigma^{2}(i, j) & =\sum_{m=1}^{j}\left(x_{m}-\mu(i, j, m)\right)^{2} \\
& =\sum_{m=1}^{j}\left(x_{m}-\beta_{i j} * m-\alpha_{i j}\right)^{2},
\end{aligned}
$$

where $\bar{t}_{i, j}=\frac{(i+j)}{2}, \bar{x}_{i, j}=\frac{\sum_{m=i}^{j} x_{m}}{j-i+1}$, and $\mu(i, j, m)=\beta_{i j} *$ $m+\alpha_{i j}, i \leq m \leq j$. It can be shown that the above equations can be rewritten into iterative forms such that can be computed in $\mathrm{O}\left(n^{2}\right)$ time for all $1 \leq i \leq j \leq n$. Due to space limitations we skip the detailed derivations here.

\section{OMSLR ALGORITHM}

We present the OMSLR algorithm for the MSLR problem as follows. Given a time series $X=\left\{x_{1}, x_{2}, \ldots, x_{n}\right\}$ and an integer $k$, the algorithm OMSLR iteratively segments the time series $X_{j}=\left\{x_{1}, x_{2}, \ldots, x_{j}\right\}$, where $1 \leq j \leq n$, into $i$ segments, starting with $i=1$ to $i=k$. Since Equation 1 is an iterative function, we design a DP algorithm to compute the matrix $M$, in which $M[i, j]=\left(\gamma_{i, j}, \rho_{i, j}^{2}\right)$, for $i=1 \rightarrow k$, as a representation of the best way of partitioning $X_{j}$ into $i$ segments $\forall j, 1 \leq j \leq n$. Here, $\gamma_{i, j}, 1 \leq \gamma_{i, j} \leq j$ denotes the starting point of the last segment of $\hat{\phi}_{i}\left(X_{j}\right)$ and the variable $\rho_{i, j}^{2}$ is the global mean square error of $i$-segment partition of $X_{j}$ based on $\hat{\phi}_{i}\left(X_{j}\right)$.

$\gamma_{i, j}$ and $\rho_{i, j}^{2}$ can be computed by the following equations.

$$
\begin{aligned}
& \gamma_{i, j}=\arg \min _{(i-1) d<m \leq j-d}\left\{\rho_{i-1, m}^{2}+\sigma^{2}(m+1, j)\right\} ; \\
& \rho_{i, j}^{2}=\rho_{i-1, \gamma_{i, j}-1}^{2}+\sigma^{2}\left(\gamma_{i, j}, j\right) .
\end{aligned}
$$

In Equation 3, the $d$ is a constant used to control the minimum size of a segmentation, the default value of $d$ is 2. With the matrix $M$ and segmentation index $\gamma_{i, j}$, we can backtrack an $i$-segment partition on $X_{j}$ denoted as $\hat{\phi}_{i}\left(X_{j}\right)=\left\{1, \hat{l}_{1}^{(i, j)}, \ldots, \hat{l}_{i}^{(i, j)}\right\}$, by the following equations.

$$
\hat{l}_{m}^{(i, j)}=\left\{\begin{array}{l}
j, m=i ; \\
\gamma_{m+1,\left(\hat{l}_{m+1}^{(i, j)}-1\right)}, 1 \leq m \leq i-1 .
\end{array}\right.
$$

In specific, the $k$-segment partition of $X_{n}$, denoted as $\hat{\phi}_{k}\left(X_{n}\right)=\left\{1, \hat{l}_{1}, \ldots, \hat{l}_{k}\right\}$, is computed as follows.

$$
\hat{l}_{i}=\left\{\begin{array}{l}
n, i=k ; \\
\gamma_{i+1,\left(\hat{l}_{i+1}-1\right)}, 1 \leq i \leq k-1,
\end{array}\right.
$$

where $x_{\hat{l}_{0}}=x_{1}$ and $x_{\hat{l}_{k}}=x_{n}$. The algorithm OMSLR, given as Algorithm 11, provides an optimal solution of the MSLR 


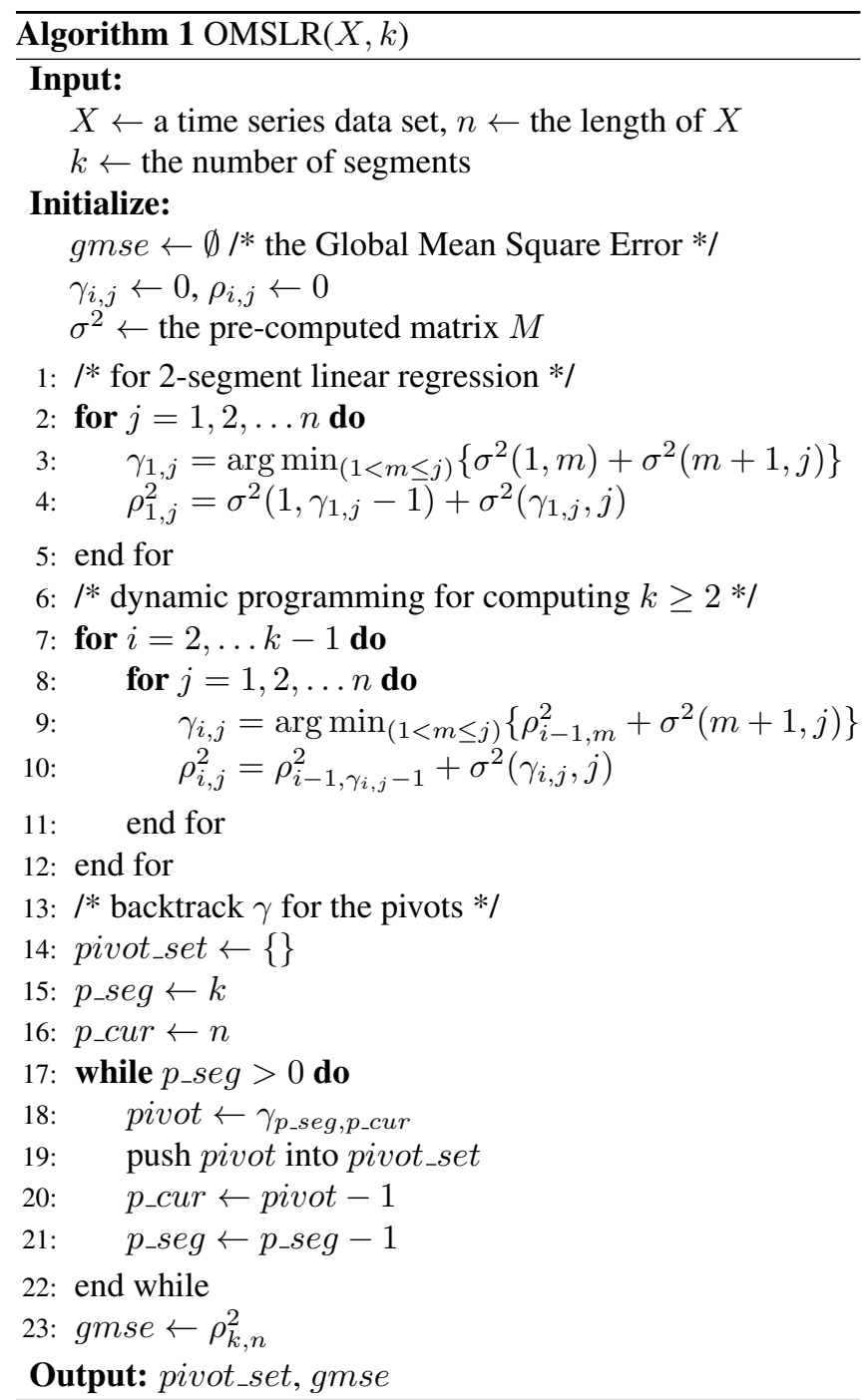

problem. In the following, we are going to show that $\hat{\phi}_{k}\left(X_{n}\right)$ is an optimal solution of the MSLR problem.

Theorem 1. Given a time series $X_{n}=\left\{x_{1}, x_{2}, \ldots, x_{n}\right\}$ and the number of segments $k$, the $i$-segment partition $\hat{\phi}_{i+1}\left(X_{j}\right), \forall j, 1 \leq j \leq n, \forall i, 1 \leq i \leq k$ as computed by Algorithm OMSLR is optimum.

Proof. We give a sketch of the proof and prove Theorem 1 by contradiction. We skip the case $k=1$, it is a natural linear regression. For the case $k=2$, it is obviously to see that $\forall j, 1 \leq j \leq n, \hat{\phi}_{2}\left(X_{j}\right)$ is optimum, since Algorithm OMSLR enumerated all the feasible solutions.

In the induction step, we assume that $\forall j, 1 \leq j \leq n$, $\hat{\phi}_{i}\left(X_{j}\right)$ is optimum. To show that $\hat{\phi}_{i+1}\left(X_{j}\right)$ is also optimum, $\forall j, 1 \leq j \leq n$, we assume that there exits an integer $\tau, 1 \leq \tau \leq n$, such that $\hat{\phi}_{i+1}\left(X_{\tau}\right)$ is not optimum. Let $\phi_{i+1}^{*}\left(X_{\tau}\right)=\left\{1, l_{1}^{*}, \ldots, l_{i}^{*}, l_{i+1}^{*}=\tau\right\}$ be the optimum $(i+1)$-segment partition of $X_{\tau}$. Then we have the following
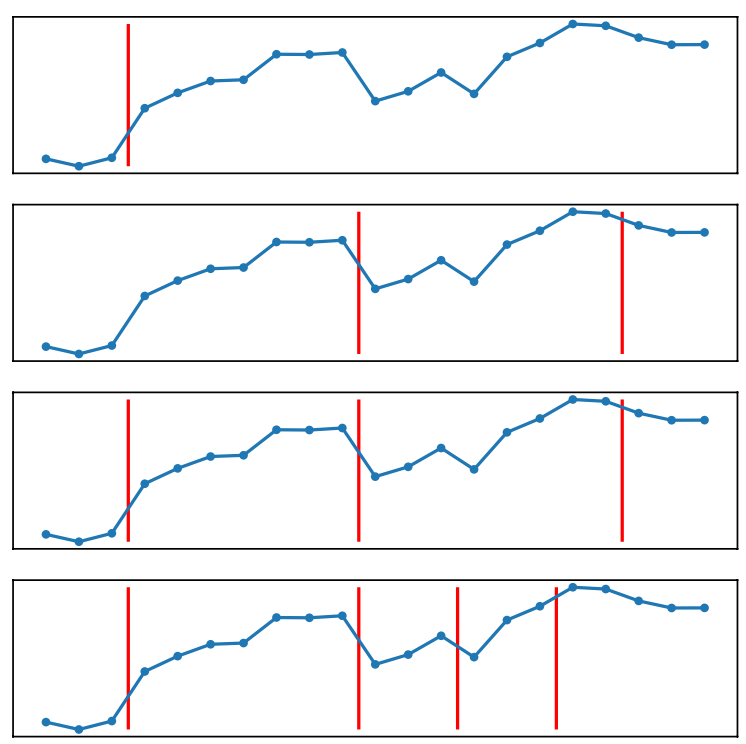

Fig. 1: A step by step results of OMSLR, for $k=2 \rightarrow 5$.

equation:

$$
\psi^{2}\left(X_{\tau} \mid \hat{\phi}_{i+1}\left(X_{\tau}\right)\right)>\psi^{2}\left(X_{\tau} \mid \phi_{i+1}^{*}\left(X_{\tau}\right)\right) .
$$

The induction assumption says that the $i$-segment partition, $\hat{\phi}_{i}\left(X_{\left.l_{i}^{*}-1\right)}\right.$ is optimum, thus it implies the Equation 5 .

$$
\psi^{2}\left(X_{l_{i}^{*}-1} \mid \phi_{i}^{*}\left(X_{l_{i}^{*}-1}\right)\right)=\psi^{2}\left(X_{l_{i}^{*}-1} \mid \hat{\phi}_{i}\left(X_{l_{i}^{*}-1}\right)\right),
$$

and Algorithm OMSLR also guarantees the Equation 6

$$
\begin{aligned}
\psi^{2}\left(X_{\tau} \mid \hat{\phi}_{i+1}\left(X_{\tau}\right)\right) & \leq \psi^{2}\left(X_{l_{i}^{*}-1} \mid \hat{\phi}_{i}\left(X_{l_{i}^{*}-1}\right)\right)+\sigma^{2}\left(l_{i}^{*}, \tau\right) \\
& =\psi^{2}\left(X_{l_{i}^{*}-1} \mid \phi_{i}^{*}\left(X_{l_{i}^{*}-1}\right)\right)+\sigma^{2}\left(l_{i}^{*}, \tau\right) \\
& =\psi^{2}\left(X_{\tau} \mid \phi_{i+1}^{*}\left(X_{\tau}\right)\right)
\end{aligned}
$$

Equation 5 and 6 imply that the assumption of Equation 4 is a contradiction. Thus we have Theorem 1 .

The running time of the algorithm OMSLR is obviously to seen to take $\mathrm{O}\left(k n^{2}\right)$ time. Due to the space limitation, we omit the detailed proof of Theorem 2

Theorem 2. The running time of the algorithm OMSLR is at most $O\left(k n^{2}\right)$ for $X_{n}=\left\{x_{1}, x_{2}, \ldots, x_{n}\right\}$ and $k$ is the number of non-overlapping segments of $X_{n}$.

To illustrate how the algorithm OMSLR handles $k$ segment partition of $X_{n}$, we show a step-by-step results in Fig. 1. To keep things simple we assume that $k=5$ and $n=22$ in this example. In the Fig. 1. each step of the $i$-segment partition is demonstrated, for $i=2 \rightarrow 5$. As shown as Theorem 11, each $i$-segment partition is an optimal result. As we presented in Section 2 and Section 3 , the first step of OMSLR algorithm is to generate the matrix $M$, which 

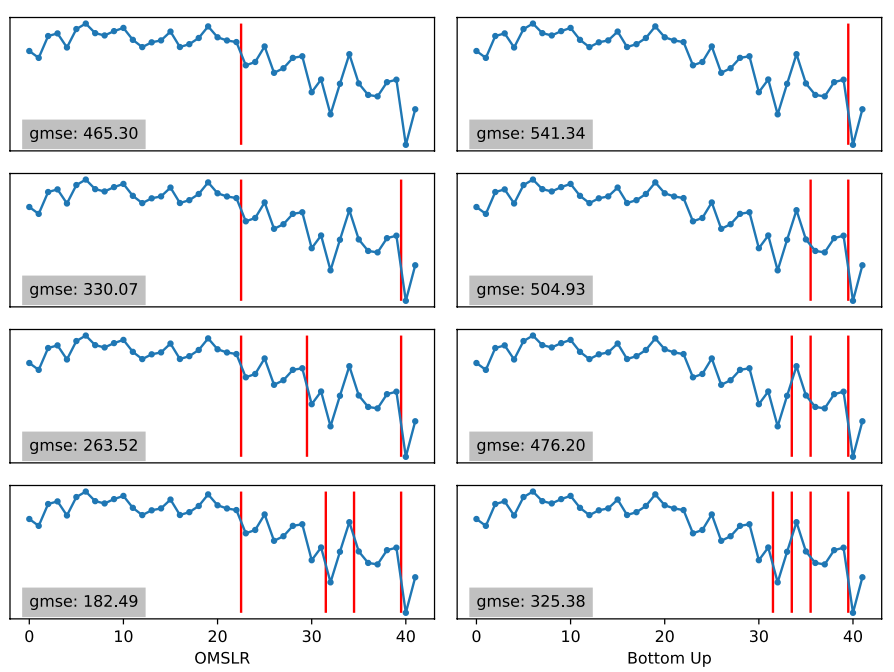

(a) The segmentations of OMSLR and Bottom-up

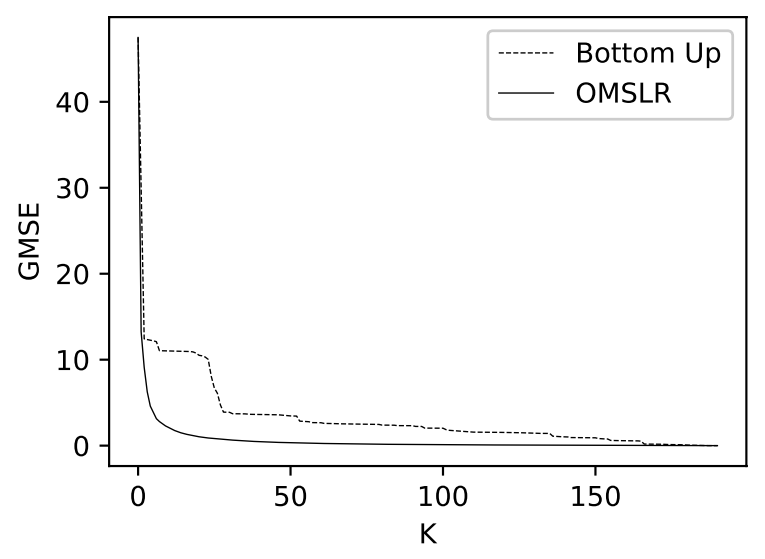

(b) The comparison of GMSE

Fig. 2: The experimental results.

can be processed iteratively based on Equation 2 in a DP way. So that the algorithm OMSLR, based on backtracking the matrix $M$, can derive each optimal $i$-segment partition by a DP approach as shown in Algorithm 1 (in Line:7 $\rightarrow$ Line:12). Algorithm 1 observably is a two-level DP design. Leveraging the two-level DP design, the algorithm OMSLR can return any $i$-segment partition of $X_{n}$ with a reasonable size of $i$ without having to re-compute from scratch. Due to its low complexity, the algorithm OMSLR offers an opportunity for one to search the best trading strategies in financial computing.

\section{EXPERIMENTAL RESULTS}

We provide an experimental evaluation of the two algorithms, i.e., our OMSLR and the Bottom-up algorithm [9], for examining the performance in terms of Global Mean Square Error
(GMSE), is defined in Section 2, with respect to the value of $k$. The two results are summarized in Fig 2 .

In the first experiment, we compare the step-by-step segment partition as $k$ varies from 2 to 5 in Fig. 2 (a). For illustration purposes, we plot the time series with small sample size. The data only contains 42 data points, and spans a period from 2008-08-01 to 2008-09-30 selected from S\&P 500 index historical daily price data. In Fig. 2(a), it is shown that OMSLR has smaller GMSE than the Bottom-up algorithm. It also shows that OMSLR always maintains optimality in partitioning the time series into multi-segment linear representation for each value of $k$.

In the second experiment, we focus on analyzing the relationship between $k$ and GMSE with a large sample size. We use S\&P 500 index historical 1-minute price data from 201007-01 to 2010-07-07 with a total of 1,560 data points. We compare the GMSE calculated by OMSLR and Bottom-up algorithm for each $k$ from 1 to 200. Fig. 22(b) demonstrates that GMSE generated by the two algorithms both decreases monotonically, and sharply at the beginning. Therefore, a searching method can be designed for locating the best value of $k$ with a given GMSE bound since the curve is a monotonically decreasing function. Compared to Bottom-up algorithm, a much smaller number of segments is required for algorithm OMSLR to find a multi-segment linear regression representation of the given time series to satisfy a given GMSE bound.

\section{CONCLUSION AND FUTURE WORK}

In this paper we study the problem of optimal segmentation of financial time series based on segmented linear regression models. We present the OMSLR algorithm based on the twolevel DP design. We show that the algorithm is optimum with time complexity $\mathrm{O}\left(k n^{2}\right)$. We also demonstrate its application in analyzing financial time series. The representation generated by the algorithm OMSLR may be fed into other intelligent applications, e.g., to predict future trend of a financial market. The algorithm may also find further applications, e.g., we may use it as a benchmark for other on-line stock trading algorithms [15]. The on-line version of the OMSLR algorithm can also be used in stock trading. We may also use the algorithm in processing data of other application domains, such as medical and data science applications. 


\section{REFERENCES}

[1] John J. Murphy, "Technical analysis of the financial markets: A comprehensive guide to trading methods and applications," New York Institute of Finance, January 1999.

[2] Ali N. Akansu, Sanjeev R. Kulkarni, and Dmitry M. Malioutov, "Financial signal processing and machine learning," Wiley-IEEE Press, May 2016.

[3] Y. Abu-Mostafa and A. F. Atiya, "Introduction to financial forecasting," Applied Intelligence, vol. 6, pp. 205-213, 2004.

[4] Victor Lavrenko, Matt Schmill, Dawn Lawrie, Paul Ogilvie, David Jensen, and James Allan, "Mining of concurrent text and time series," in Proceedings of 6th ACM SIGKDD Workshop on Text Mining. 2000, pp. 3744, ACM.

[5] Ella Bingham, Aristides Gionis, Niina Haiminen, Heli Hiisilä, Heikki Mannila, and Evimaria Terzi, "Segmentation and dimensionality reduction," in Proceedings of the 2006 SIAM International Conference on Data Mining, pp. 372-383.

[6] Jessica Lin, Eamonn Keogh, Li Wei, and Stefano Lonardi, "Experiencing sax: a novel symbolic representation of time series," Data Mining and Knowledge Discovery, vol. 15, no. 2, pp. 107-144, 2007.

[7] Philippe Esling and Carlos Agon, "Time-series data mining," ACM Comput. Surv., vol. 45, no. 1, Dec. 2012.

[8] Richard Bellman, "On the approximation of curves by line segments using dynamic programming," Commun. ACM, vol. 4, no. 6, pp. 284, June 1961.

[9] Eamonn Keogh, Selina Chu, David Hart, and Michael Pazzani, "Segmenting time series: A survey and novel approach," in Data Mining in Time Series Databases, 2004, pp. 1-21.

[10] H. Shatkay and S. B. Zdonik, "Approximate queries and representations for large data sequences," in Proceedings of the Twelfth International Conference on Data Engineering, 1996, pp. 536-545.

[11] Guy Rosman, Mikhail Volkov, Danny Feldman, John W. Fisher III, and Daniela Rus, "Coresets for ksegmentation of streaming data," in Proceedings of the 27th International Conference on Neural Information Processing Systems - Volume 1, Cambridge, MA, USA, 2014, NIPS'14, p. 559-567, MIT Press.

[12] Jayadev Acharya, Ilias Diakonikolas, Jerry Li, and Ludwig Schmidt, "Fast algorithms for segmented regression," in Proceedings of the 33rd International Conference on International Conference on Machine Learning - Volume 48. 2016, ICML'16, p. 2878-2886, JMLR.org.

[13] Evimaria Terzi and Panayiotis Tsaparas, "Efficient algorithms for sequence segmentation," in Proceedings of the Sixth SIAM International Conference on Data Mining, 2006.

[14] Eamonn J. Keogh and Michael J. Pazzani, "An enhanced representation of time series which allows fast and accurate classification, clustering and relevance feedback," in Proceedings of the Fourth International Conference on Knowledge Discovery and Data Mining. 1998, p. 239-243, AAAI Press.

[15] L. Conegundes and A. C. M. Pereira, "Beating the stock market with a deep reinforcement learning day trading system," in 2020 International Joint Conference on Neural Networks (IJCNN), 2020, pp. 1-8. 